VOL. $3(1970), 75-79$.

\title{
A commutativity theorem for power-associative rings
}

\section{L. Outcalt and Adil Yaqub}

\begin{abstract}
Let $R$ be a power-associative ring with identity and let $I$ be on ideal of $R$ such that $R / I$ is a finite field and $x \equiv y$ $(\bmod I)$ implies $x^{2}=y^{2}$ or both $x$ and $y$ commute with all elements of $I$. It is proven that $R$ must then be commutative. Examples are given to show that $R$ need not be commutative if various parts of the hypothesis are dropped or if $" x^{2}=y^{2 "}$ is replaced by " $x^{k}=y^{k}$ " for any integer $k>2$.
\end{abstract}

\section{Introduction}

Wedderburn's Theorem, asserting that a finite associative division ring is necessarily commutative, has recently been generalized by the authors in $[1 ; 2]$. Indeed, the following theorem, the case $N=(0)$ of which yields Wedderburn's Theorem, was proved in [2]:

THEOREM 1. Let $R$ be an associative ring with identity in which every element is either nilpotent or a unit in $R$. Then

(a) the set $N$ of nilpotent elements in $R$ is an ideal and $R / N$ is a division ring;

(b) if (i) $R / N$ is finite, and (ii) $x \equiv y(\bmod N)$ implies $x^{2}=y^{2}$ or both $x$ and $y$ commute with all elements of $N$, then $R$ is commutative.

Our present object is to extend Theorem 1 to the case where $R$ is a

Received 12 April 1970. The first author was supported by US Air Force Office of Scientific Research Grant No. 698-67, and the second author by US National Science Foundation Grant GP-5929. 
power-associative ring and where $I$ is a more general ideal in $R$ than $N$. Indeed, we prove the following

THEOREM 2. Let $R$ be a power-associative ring with identity 1 , and let $I$ be an ideal in $R$. If, further,

(i) $R / I$ is a finite field, and

(ii) $x \equiv y(\bmod I)$ implies $x^{2}=y^{2}$ or both $x$ and $y$ commute with all elements of $I$,

then $R$ is conmutative.

We also give examples to show that Theorem 2 need not be true if either hypothesis (i) or (ii) is dropped, or if the hypothesis that $R$ has an identity is deleted. Moreover, it turns out, somewhat surprisingly perhaps, that this theorem is not necessarily true if $" x^{2}=y^{2 \prime}$ in (ii) is replaced by $" x^{k}=y^{k_{n}}$ for any $k>2$ (see examples below).

\section{Main section}

Proof of Theorem 2. First, we prove that $I$ is commutative. Suppose that $a_{1}, a_{2} \in I$ and $a_{1} a_{2} \neq a_{2} a_{1}$. We shall show that this Ieads to a contradiction. Since $a_{1} \equiv 0(\bmod I), a_{2} \equiv 0(\bmod I)$, $a_{1}+a_{2} \equiv 0(\bmod I)$, and $a_{1} a_{2} \neq a_{2} a_{1}$, we have by (ii),

$$
a_{1}^{2}=0, a_{2}^{2}=0,\left(a_{1}+a_{2}\right)^{2}=0 \text {. }
$$

Hence, $a_{1} a_{2}+a_{2} a_{1}=0$. Moreover, since $a_{1}+1 \equiv 1(\bmod I)$ and $\left(a_{1}+1\right) a_{2} \neq a_{2}\left(a_{1}+1\right)$, we have using (ii) again, $\left(a_{1}+1\right)^{2}=1$. Hence, since $a_{1}^{2}=0,2 a_{1}=0$. Therefore

$$
a_{1} a_{2}=-a_{2} a_{1}=a_{2} a_{1} \text {, }
$$

and thus $I$ is indeed commutative.

Now, suppose $a \in I$ and $b \in R$. We shall show that $a b=b a$. Suppose not. Since $a+b \equiv b \quad(\bmod I)$ and $a b \neq b a$, we have by (ii), $(a+b)^{2}=b^{2}$ and hence $a^{2}+a b+b a=0$. Since, moreover, $-a+b \equiv b$ $(\bmod I)$, a similar argument shows that $a^{2}-a b-b a=0$. Hence, upon subtracting, we get $2(a b+b a)=0$. Moreover, since $a b \neq b a$, $a(b+1) \neq(b+1) \alpha$, and hence we may repeat the above argurent using $b+1$ 
instead of $b$ to get $2(a(b+1)+(b+1) a)=0$. Combining this equation with $2(a b+b a)=0$, we get $4 a=0$ and hence $-2 a=2 a$. Thus $2 a b=-2 b a=2 b a$, and hence

$$
2(a b-b a)=0 \text {. }
$$

Now, let $p$ be the characteristic of the finite field $R / I$ (see (i) : Then $p b \in I$, and hence $a(p b)=(p b) a$. Therefore

$$
p(a b-b a)=0 \text {. }
$$

We now distinguish two cases.

Case 1. $p \neq 2$. Then $p$ is an odd prime and (1), (2) readily imply $a b-b a=0$, a contradiction.

Case 2. $p=2$. In this case the finite field $R / I$ has exactly $2^{k}$ elements for some integer $k$. Hence $(\bar{b})^{2^{k}}=\bar{b}$, and thus $b^{2^{k}}-b \in I$. Therefore,

$$
a\left(b^{2^{k}}-b\right)=\left(b^{2^{k}}-b\right) a \text {. }
$$

Moreover, since $(a+b)^{2}=b^{2}$ and $R$ is power-associative, we obtain $\left\{(a+b)^{2}\right\}^{2^{k-1}}=\left(b^{2}\right)^{2^{k-1}}$, hence $(a+b)^{2^{k}}=b^{2^{k}}$. Now, by the power-associativity of $R,(a+b)(a+b)^{2^{k}}=(a+b)^{2^{k}}(a+b)$, therefore $(a+b) b^{2^{k}}=b^{2^{k}}(a+b)$. Thus, using power-associativity again, we get

$$
a b^{2^{k}}=b^{2^{k}} a
$$

Combining (3) and (4), we get $a b=b a$, a contradiction. We have thus obtained a contradiction whether $p \neq 2$ or $p=2$. This contradiction proves that

$$
a b=b a \text { for all } a \in I \text { and all } b \in R \text {. }
$$

To complete the proof of the theorem, suppose $x, y \in R$. In view of (5), we may assume that $x \notin I$ and $y \notin I$. Let $\xi=\xi+I$ be a generator for the multiplicative cyclic group of non-zero elements of the finite field $R / I$. Then for some integers $i, j$, and some elements $a, a^{\prime} \in I$, we have,

$$
x=\xi^{i}+a, y=\xi^{j}+a^{\prime}
$$


Hence, by (5), the power-associativity of $R$, and the fact (proved above) that $I$ is commutative, we readily obtain that $x y=y x$. This proves the theorem.

\section{Examples and remarks}

In this section, we give some examples to show that Theorem 2 need not be true if either hypothesis (i), (ii) is deleted, or if the hypothesis that $R$ has an identity is dropped.

EXAMPLE 1. Let $R$ be the ring of quaternions, and let $I=(0)$. Here $R$ satisfies ( $i i)$, but (i) fails to hold. Another example is furnished by taking $R$ to be the complete matrix ring, $M_{n}(F)$, over a field $F$, and $I=(0)$. Clearly both of these rings are not commutative.

EXAMPLE 2. Let

$$
\begin{aligned}
& R=\left\{\left[\begin{array}{lll}
a & b & c \\
0 & a & d \\
0 & 0 & a
\end{array}\right] \mid a, b, c, d \in G F(2)\right\}, \\
& I=\left\{\left[\begin{array}{lll}
0 & b & c \\
0 & 0 & d \\
0 & 0 & 0
\end{array}\right] \mid b, c, d \in G F(2)\right\} .
\end{aligned}
$$

It is readily verified that $R$ satisfies ( $i$ ), but (ii) fails to hold. Moreover, $R$ is not commutative.

EXAMPLE 3. Let

$$
\begin{aligned}
& R=G F(q) \oplus L, \\
& I=L,
\end{aligned}
$$

where $L$ is a Lie ring of characteristic not 2 . Then $R$ satisfies all the hypotheses of Theorem 2, except that $R$ has no identity 1 . Moreover, $R$ is not commutative.

We now remark that the equation " $x^{2}=y^{2 "}$ in (ii) of Theorem 2 cannot in general be replaced by $" x^{k}=y^{k}$ for any $k>2$. For, consider the ring $R$ defined by 


$$
R=\left\{\left[\begin{array}{lll}
a & b & c \\
0 & a & d \\
0 & 0 & a
\end{array}\right] \mid a, b, c, d \in G F(p), p=\text { prime }\right\},
$$

where $p$ is chosen, in two stages, as follows: if $k$ is odd, take $p$ to be any fixed prime divisor of $k$; while, if $k$ is even, take $p$ to be any fixed prime divisor of $k / 2$. Since $k>2$, such a prime $p$ always exists. Let

$$
I=\left\{\left[\begin{array}{lll}
0 & b & c \\
0 & 0 & d \\
0 & 0 & 0
\end{array}\right] \mid b, c, d \in G F(p)\right\} .
$$

It is easily seen that $R$ satisfies all the hypotheses of Theorem 2, except that $" x^{2}=y^{2 "}$ is now replaced by $" x^{k}=y^{k}$ in (ii). However, $R$ is not commutative.

Now, if in Theorem 2, we specialize $R$ to be an associative ring with identity such that every element in $R$ is either nilpotent or a unit in $R$, then it is easily seen that the set $N$ of nilpotent elements in $R$ forms an ideal, and that $R / N$ is indeed an associative division ring. If, in addition, $R / N$ is finite, then $R / N$ is a field (by Wedderburn's Theorem), and Theorem 1 now follows at once from Theorem 2 upon specializing the ideal $I$ to be $N$ itself.

Whether or not the assumption of power-associativity in Theorem 2 is essential remains an open question.

\section{References}

[1] D.L. Outcalt and Adil Yaqub, "A generalization of Wedderburn's theorem", Proc. Amer. Math. Soc. 18 (1967), 175-177.

[2] D.L. Outcalt and Adil Yaqub, "A commutativity theorem for rings", Bull. Austral. Math. Soc. 2 (1970), 95-99.

University of Cal ifornia, Santa Barbara, California. 